\title{
$\widehat{A}$ Madridge \\ madridge Journal of Nursing \\ Interconnecting Scientific World
}

Research Article

Open Access

\section{Assess the effect of Cardiopulmonary Resuscitation Demonstration on Knowledge, Attitude and Practice among Relatives of Cardiac Patients}

\author{
Viji $\mathbf{P}^{1}$, and Santra Baby ${ }^{2 *}$ \\ 'Professor, KMCH College of Nursing, Coimbatore, Tamil Nadu, India \\ ${ }^{2}$ GKNM Institute of Nursing, Coimbatore, Tamil Nadu, India
}

\section{Article Info \\ *Corresponding author: \\ Santra Baby \\ GKNM Institute of Nursing \\ Coimbatore \\ Tamil Nadu \\ India \\ E-mail: santrababys@gmail.com}

Received: July 23, 2018

Accepted: August 8, 2018

Published: August 13, 2018

Citation: Viji P, Baby S. Assess the effect of Cardiopulmonary Resuscitation Demonstration on Knowledge, Attitude and Practice among Relatives of Cardiac Patients. Madridge J Nurs. 2018; 3(2): 124-126.

doi: 10.18689/mjn-1000122

Copyright: ( $₫ 2018$ The Author(s). This work is licensed under a Creative Commons Attribution 4.0 International License, which permits unrestricted use, distribution, and reproduction in any medium, provided the original work is properly cited.

Published by Madridge Publishers

\begin{abstract}
Cardiopulmonary resuscitation (CPR) is an emergency procedure that combines chest compressions often with artificial ventilation in an effort to manually preserve intact brain function until further measures are taken to restore spontaneous blood circulation and breathing in a person who is in cardiac arrest. The families of those who survive a heart attack or have a history of heart problems should also take CPR training. Chance of survival increases four-fold with immediate action. Hence, a quasi experimental research was conducted to assess the effect of cardiopulmonary resuscitation demonstration on knowledge, attitude and practice among relatives of cardiac patients of tertiary care hospital, south India. Totally 30 subjects who fulfilled the criteria were chosen by convenient sampling technique. Conceptual framework comprised of Ludwing Bertalanffy's General System Theory. Semi structured interview questionnaire was utilized to assess the knowledge. Attitude was assessed through the scale and the practice was measured using the checklist. Subjects were tested both before and after cardiopulmonary resuscitation demonstration. The mean difference of knowledge was 9.73 , with combined $S D$ of 5.51 and ' $t$ ' value of 15.35 . Paired' $t$ ' test showed that there was a significant difference in pre-test and post-test level of knowledge at 0.05 level. The calculated attitude't' value (5.56) and practice' $t{ }^{\prime}$ value (85.57) were higher than table values. The study findings highlighted the advantages of having demonstration in improving knowledge, attitude and practice of cardiopulmonary resuscitation among relatives of cardiac patients.
\end{abstract}

Abbreviations: CPR: Cardio Pulmonary Resuscitation.

Keywords: CPR demonstration; Knowledge; Attitude; Practice; Cardiac patients; Relatives.

\section{Introduction}

Human heart beats about 1,00,000 times a day, 70-80 times a minute, surges 2000 gallons of blood through 60,000 miles of blood vessels which feeds the body organs and tissues. Heart receives its blood supply from the right and left coronary arteries which originates from aorta [1].

Any damage to the coronary artery reduces the pumping power of heart. When the heart fails to contract effectively; cardiac arrest occurs. Arrested blood circulation prevents delivery of oxygen to the body. Lack of oxygen to the brain causes loss of consciousness, which results in abnormal or absent breathing. Cardiac arrest if untreated can damage brain or can lead to a sudden death [2].

Cardiopulmonary resuscitation is a lifesaving technique useful in many emergencies, including heart attack or near drowning. It consists of the use of chest compressions 
and artificial ventilation to maintain circulatory flow and oxygenation during cardiac arrest [3]. Although survival rates and neurologic outcomes are poor for patients with cardiac arrest, appropriate resuscitation involving early defibrillation and proper implementation of post cardiac arrest care lead to improved survival and neurologic outcomes.

Indians are far behind in the global surge to learn cardiopulmonary resuscitation. The World Heart Federation reports that less than $1 \%$ Indians would presently know how to carry out a cardiopulmonary resuscitation. Being trained to perform cardiopulmonary resuscitation can make the difference between life and death [4]. The proffered way to learn cardiopulmonary resuscitation is to practice cardiopulmonary resuscitation. Therefore the present study was conducted with the following objectives:

- To assess the level of knowledge, attitude and practice regarding cardiopulmonary resuscitation among relatives of cardiac patients.

- To assess the effectiveness of cardiopulmonary resuscitation demonstration among relatives of cardiac patients.

- To determine the association between knowledge, attitude and practice with selected demographic variables.

\section{Methods}

\section{Study Sample and setting}

A quasi experimental research design was adopted. Ludwing Bertalanffy's General system theory was adopted for conceptual framework. Quasi pre experimental one group pretest posttest design was selected for this study. Eligibility criteria included relatives of cardiac patients waited in cardiac wards, were willing to re-demonstrate cardiopulmonary resuscitation procedure and understood Tamil or English were included in the study. Relatives who were not competent to perform CPR and may not be with patient on an emergency were excluded from sampling. Ethical protocols and hospital's policy on protection of patients' rights, privacy and confidentiality were assured firmly. The authors provided patient and their relatives with oral and written clarifications about the purpose of the study and its procedures and asked for their willingness to participate in the study. After obtaining patients and relatives' consent, Tamil versions of knowledge questionnaire, attitude scale and practice checklist were administered to each participant during a face-to-face interview. The English version of the used tools was forward and backward translated into Tamil by independent expert and underwent pilot testing with representative homogenous sample. After completing data collected packages were kept safely to guarantee confidentiality and anonymity.

\section{Tools}

\section{Knowledge questionnaire}

The knowledge items had 20 questions. The correct answer was scored as ' 1 ' and the wrong answer as ' 0 '.the scores were interpreted as 0-6: Poor knowledge, 7-13: Fair knowledge, 14-20: Good knowledge.

\section{Attitude scale}

Attitude scale had ten statements, with five positive and five negative scores. The scores $<50 \%$ were considered as unfavourable, $50-75 \%$ : moderate and $>75 \%$ was scored as favourable.

\section{Practice checklist}

Practice checklist had 15 steps.Score ' 1 ' was given for each right performance. ' 0 ' score was given if the steps went wrong. The ranges were interpreted as $<4$ score, poor performance, 4-7 moderate, 8-11 good and 11-15 for excellent performance.

\section{Data Analysis}

Data analyses were conducted using the IBM SPSS 20.0. Descriptive statistics were used to describe the demographics of the sample. Mean and standard deviations were used to describe the distributions of knowledge, attitude and practice on CPR. Paired " $\mathrm{t}$ " test was used to compare the pre and post test results.

\section{Results}

\section{Subject Characteristics}

A total of 30 relatives of patients waited near intensive coronary care unit and in cardiology ward were approached to participate in the study $[9,10]$. Most of the participants belonged to the non medical profession (97\%). Majority had previous knowledge of CPR (83\%) and almost everyone had never seen cardiopulmonary resuscitation procedure (97\%).

In pre-test majority samples (53\%) had fair knowledge whereas in post test most (90\%) of the study participants had good knowledge [10]. Similarly in pre-test majority (57\%) had moderate and (53\%) had favourable attitude towards CPR in post-test.

Figure 1. Number of relative's vs Levels of practice

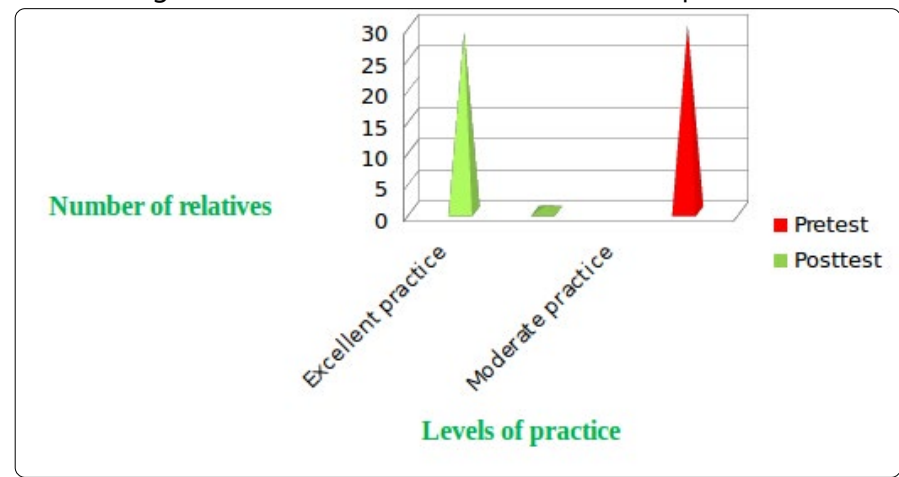

All participants (100\%) had poor practices when pretested. This figured (97\%) excellence after intervention, with the mean score 13.7 and a SD of 0.86 .

Table 1. Comparison of mean pre-test/post-test levels of knowledge on CPR

\begin{tabular}{|l|l|l|l|l|l|}
\hline Group & Mean of differences & Combined SD & ' $t$ ' value & $\mathrm{df}$ & Table value \\
\hline Pre test & 9.73 & 5.51 & $15.35^{\star}$ & 29 & 1.699 \\
\hline Post test & & & & \\
\hline
\end{tabular}

* Level of significance-0.05 
Table 2. Comparison of mean pre-test/post-test levels of attitude on CPR

\begin{tabular}{|l|l|l|l|l|l|}
\hline Group & Mean of differences & Combined SD & ' $t$ ' value & df & Table value \\
\hline Pre test & 10.1 & 11.38 & 5.56 & 29 & 1.699 \\
\hline Post test & & & & \\
\hline
\end{tabular}

*Level of significance-0.05

Table 3. Comparison of mean pre-test/post-test levels of practice on CPR

\begin{tabular}{|l|l|l|l|l|l|}
\hline Group & Mean of differences & Combined SD & 't' value & $\mathrm{df}$ & Table value \\
\hline Pre test & 13.7 & 0.86 & 85.57 & 29 & 1.699 \\
\hline Post test & & & & \\
\hline
\end{tabular}

*Level of significance-0.05

\section{Reliability assessment}

The reliability of the tool was determined by the Spearman Brown's Split-Half technique showing $r=0.89$ for knowledge questionnaire, $r=0.99$ for attitude scale and $r=0.98$ for practice checklist. Hence the tool was found highly reliable.

\section{Validity assessment}

The content validity of the instrument was ensured based on opinion from experts.

\section{Discussion}

Present study findings were consistent with Rajpakse (2010) [5] who assessed the knowledge of public regarding cardiopulmonary resuscitation. Telephonic survey regarding knowledge on cardiopulmonary resuscitation revealed that trained people had better knowledge on cardiopulmonary resuscitation than untrained people even though both of them had low knowledge levels.

Also current study correlates the evidence by Reder $\mathrm{S}$ (2006) [6] who reported that hands on training on cardiopulmonary resuscitation were effective in improving knowledge and performance of cardiopulmonary resuscitation among school students [7].

The mean difference of attitude was 10.1, with the combined SD of 11.38, hence found significant difference in pre-test and post-test attitude scores [8]. Similarly the mean difference of practice scores was 13.7, with the combined SD of 0.86 , and found significance difference in post-test.

Hypotheses were verified and gave rise to positive correlations.

\section{Conclusion}

Thus the study concluded that, demonstration on cardiopulmonary resuscitation was an eminent and effective method to improve knowledge, attitude and practice regarding cardiopulmonary resuscitation. Demonstration on cardiopulmonary resuscitation can be introduced as a routine program for relatives of cardiac patients in clinical areas. Nursing research on cardiopulmonary resuscitation would be a valuable reference material for further researchers.

\section{References}

1. Siddhique A. Effect of Vasodilation and Arterial Resistance on Cardiac Output. Journal of Clinical and Experimental Cardiology. 2011; 2(11). doi: 10.4172/2155-9880.1000170

2. Modi S, Krahn AD. Sudden Cardiac Arrest without Overt Heart Disease. Circulation Journal. 2011; 123(25): 2994-3008. doi: 10.1161/ CIRCULATIONAHA.110.981381

3. Chandra N, Bastiaenen R, Papadakis M. Sudden Cardiac Death In Young Athletes: Practical Challemnges \& Diagnostic dilemmas. Journal of the American college of cardiology. 2013; 61(10): 1027-1038. doi: 10.1016/j. jacc.2012.08.1032

4. Lidhoo P. Evaluating the effectiveness of CPR for in hospital cardiac arrest. American Journal of Hospice and Palliative care. 2012; 30(3): 279-282. doi: 10.1177/1049909112448522

5. Rajapakse R, Noč $M$, Kersnik J. Public knowledge of cardiopulmonary resuscitation in Republic of Slovenia. The Central European Journal of Medicine. 2010; 122(23): 667-72. doi: 10.1007/s00508-010-1489-8

6. Reder S, Cummings P, Quan L. Comparison of three instructional methods for teaching cardiopulmonary resuscitation and use of an automatic external defibrillator to high school students. Resuscitation Journal. 2006; 69(3): 443-53. doi: 10.1016/j.resuscitation.2005.08.020

7. Savastano S, Vanini V. Cardiopulmonary resuscitation in real life: the most frequent fears of lay rescuers. Resuscitation Journal. 2011; 82(5): 568-71. doi: 10.1016/j.resuscitation.2010.12.010

8. Suto T, Saito $S$. Considerations for resuscitation at high altitude in elderly and untrained populations and rescuers. The American Journal of Emergency Medicine. 2014; 32(3): 270-6. doi: 10.1016/j.ajem.2013.11.007

9. Taniguchi $\mathrm{T}, \mathrm{Omi} \mathrm{W}$, Inaba $\mathrm{H}$. Attitudes toward the performance of bystander cardiopulmonary resuscitation in Japan. Resuscitation Journal. 2007; 75(1): 82-7. doi: 10.1016/j.resuscitation.2007.02.019

10. Takei $Y$, Nishi $T$, Matsubara $H$, Hashimoto $M$, Inaba $H$. Factors associated with quality of bystander CPR: the presence of multiple rescuers and bystander-initiated CPR without instruction. Resuscitation. 2014; 85(4): 492-8. doi: 10.1016/j.resuscitation.2013.12.019 